\title{
An analogy to understand the conceptual foundations of electromagnetics at the micro level from the perspective of mechanics
}

Wei Fan ( $\nabla$ wfan10@163.com )

Institute of Theoretical Physics

\section{Research Article}

Keywords: charge, current, electric field, magnetic field, Maxwell

Posted Date: February 17th, 2021

DOl: https://doi.org/10.21203/rs.3.rs-251680/v1

License: (c) (1) This work is licensed under a Creative Commons Attribution 4.0 International License.

Read Full License 


\title{
An analogy to understand the conceptual foundations of electromagnetics at the micro level from the perspective of mechanics
}

Wei Fan*

Institute of Theoretical Physics, Yunnan, Kunming, 650000 P. R. China

E-mail:wfan10@163.com

\begin{abstract}
This article attempts to provide a new analogy understanding of some conceptual foundations of electromagnetics from the perspective of mechanics to satisfy childhood curiosity. Among them, from the perspective of mechanics, charge can be understood as a form of electron momentum; electric current can be understood as a momentum flow; resistance can be understood as a momentum resistance of electrons; voltage can be interpreted as the potential pressure or energy level difference of the electron orbital potential; the electric field can be understood as a manifestation of the magnetic field of the current element.
\end{abstract}

Keywords charge; current; electric field; magnetic field; Maxwell.

\section{Introduction}

When I was about four or five years old, I liked to look up at the stars and the moon quietly by myself, and wondered how the stars and the moon float in the sky, and why don't they fall? In addition, I particularly like playing with magnets, and I also like to use rubbed rulers to attract scraps of paper for fun. This is why I later engaged in physics research and wrote this thesis. In the later study and research, many problems gradually grew in my heart. Such as, what is the origin of charge on the micro level? What is the origin of electric current at the micro level? What is the origin of voltage at the micro level? What is the origin of resistance at the micro level? What is the origin of the electric field at the micro level? What is the origin of the magnetic field at the micro level? What is the origin of electromagnetic force at the micro level? What is the origin of electromagnetic waves at the micro level? What is the origin of magnetic monopole on the microscopic level? This has always been the mystery of physics. At the same time, looking at the relevant literature, we can understand that predecessors have made a lot of efforts in these fields [1-13]. Therefore, I also want to contribute in my own way.

Among them, I gained a new understanding of current and voltage by interpreting the origin of electric charge on a microscopic level from the perspective of mechanics. At the same time, I also interpret the origins of electric and magnetic fields from the perspective of mechanics at the micro level to obtain a feasible way to explain the properties of the electric field with the properties of the magnetic field.

\section{New understanding of charge, current and voltage from the perspective of mechanics}

According to the classical electromagnetic theory [14], the conduction current is the quantity of charges that flow across a section of a conductor per unit time, which can be expressed mathematically as follows: 


$$
I=\frac{d q}{d t}
$$

Since the conduction current is based on the Drude-Lorentz model and Drude-Sommerfeld model [15]. Among them, free electrons transfer charges through classical collisions to generate electric current. From the perspective of classical mechanics, the electron collision should transfer the momentum of the electron. Therefore, from the perspective of mechanics, we can think that the charge transferred in the conduction current should be a manifestation of electron momentum, and the current should be a momentum flow.

In addition, based on the new understanding of atomic physics [16] and quantum mechanics [17] at the micro level, especially according to the photoelectric effect, the energy level transition of electrons can generate electric current. In other words, electrons generate electric current by transferring energy specific to the energy level difference. Considering that the energy of the energy level difference of electrons is a manifestation of electron momentum, that is to say, at the microscopic level, the essence of electron transfer charge to generate current is the electron transfer momentum to generate momentum flow. In other words, from the perspective of mechanics, charge is a manifestation of electron momentum, and current is a momentum flow. That is to say, the current is the transfer of momentum $P$ transfer between electrons. Because electrons movement around the nucleus, so here the electronic momentum $P$ is the electronic angular momentum increment or impulse $u$, namely $P \Rightarrow u$. The current $I$ can be expressed as the quantity of electronic angular momentum increment or impulse $u$ that flow across a section of a conductor per unit time. Thus, the origin of the charge is the increase or impulse of the electron angular momentum $u$. Then, conduction current can become conduction Momentum flow $I \Rightarrow I_{p}$ :

$$
I \Rightarrow I_{p}=\frac{d p}{d t}=\frac{d u}{d t}
$$

Of course, from the above formula, we can see that from a mechanical point of view that current is a momentum flow, and the charge in current is a form of electron momentum. Therefore, we do not need to consider the Coulomb force between electrons and electrons in the momentum flow. The electrons can be paired to form momentum flow through direct collisions to transfer momentum, and there is no need to introduce pairing or coupling media. such, when the momentum flow has no momentum loss during the transmission process, the transmission process will become an ideal superconductor. Therefore, the above new understanding of current from the perspective of mechanics can also provide a new theoretical explanation for the phenomenon of low-temperature superconductivity and high-temperature superconductivity at the micro level.

Then, the momentum flow density can be expressed as:

$$
J_{p}=\frac{d p}{d t \cdot d s_{\perp}}
$$

If the number of electrons per unit volume in the conductor is $n$, then $\rho=p n$ ( $p$ is electron momentum). Then the momentum flow density in the conductor is:

$$
J_{p}=n p v
$$


The relationship between the momentum flow density and momentum flow in the surface $s$ is:

$$
I_{p}=\iint_{s} j_{p} \cdot d s
$$

On closed surfaces, it is:

$$
I_{p}=\oiint_{s} j_{p} \cdot d s
$$

The continuity equation of its momentum flow is:

$$
I_{p}=\oiint_{s} j_{p} \cdot d s=-\frac{d p_{\text {int }}}{d t}
$$

Because the current is the momentum flow, so, the electromotive force is the impulse force of the electron, expressed as:

$$
\varepsilon=\int \frac{d p}{d t} d t
$$

Because from the mechanical point of view, the current is the momentum current produced by the impulse of the electron angular momentum. So, from the mechanical point of view, the voltage is the orbital potential difference or energy level difference or potential pressure:

$$
U_{a b}=E_{a}-E_{b}=\varepsilon
$$

Therefore, I gave a new definition of voltage at the micro level from the perspective of mechanics, and prove that the origin of voltage is a potential pressure (potential difference or energy level difference).

Considering that current is momentum flow from a mechanical point of view, resistance can be regarded as a momentum resistance $R \Rightarrow R_{p}$.

Summary Through the above derivation, we can see that based on the new understanding of atomic physics and quantum mechanics at the microscopic level in the 20th century. At the micro level, from the perspective of mechanics, we should understand current as momentum flow, and electrons generate current by transferring momentum; charge should be understood as a manifestation of electronic momentum, specifically in the form of electronic momentum or the increment (impulse) of electronic angular momentum; voltage should be understood as the potential difference (energy level difference) of electrons. Thus, we have proposed a new understanding of current, charge and voltage at the micro level from the perspective of mechanics.

3 New understanding of electric field, magnetic field, electromagnetic force and electromagnetic wave at the micro level from the perspective of mechanics

Based on the new cognition of atomic physics, we know the atomic model, know the existence of electrons, know the electron spin, know the movement of electrons around the nucleus, and know the existence of electron magnetic moments. According to the classical electromagnetic theory, the electric field intensity is expressed as:

$$
E=\frac{F}{q}
$$


According to the above, from the perspective of mechanics, the nature of the charge is the impulse $u$ of the electronic angular momentum. According to classical electromagnetic theory and quantum optics theory [18], the electric field is generated by the charge, and the photon is the medium particle of the electric field. It follows that the electric field force is the force produced by the collision of electronic angular momentum impulses with photons. This force is an impulse moment, expressed as $M=r \times u$. The impulse of the electronic angular momentum is generated by electron spin and orbital motion, so, the field source of the electric field is the electron magnetic moment, and the field source particles are electrons $e$, namely $q \Rightarrow e$. Given that the magnetic field

is generated by the molecular magnetic moment $\sum m \neq 0$, while the magnetic moment is generated by the electron spin angular momentum and orbital angular momentum, and also given that $\mathrm{t}$ the photon is the medium particles of the magnetic field, then the magnetic field force can also be seen as the force produced by the collision of electronic angular momentum impulses with photons. This force is also an impulse moment, expressed as $M=r \times u$. Accordingly, the field source of the magnetic field is also the electron magnetic moment, and the field source particles are also electrons $e$. Therefore, we can deduce that the electric field $E$ and the magnetic field $H$ have the same field source, which is the electron $e$. So, we can get a powerful equation that explains that a magnetic field and an electric field have the same field source:

$$
\left\{\begin{array}{l}
H=\frac{F}{e} \\
E=\frac{F}{e}
\end{array}\right.
$$

According to the above derivation, from the perspective of mechanics, since the electric field and the magnetic field have the same field source, the electric field and the magnetic field belong to the same object. This signifies that the nature of the electric field is the magnetic field, so the nature of the electric field force is the magnetic force and the acting force of the positive and negative charges is the acting force of the two magnetic poles. Therefore, we explain the electric field from the aspect of the magnetic field, and explain the acting force of positive and negative charges from the aspect of the magnetic force. Electromagnetic waves can be interpreted as media fluctuations generated by electronic collision media. The amount of charge measured by Millikan's 'oil-drop' experiment can be interpreted as the amount of action produced by the electron magnetic moment. The positive and negative charges can be interpreted as the behavior of the two magnetic poles of the magnetic field. (Note: This is a new perspective on electromagnetics based on the new cognition of atomic physics obtained at the micro level in the 20th century. Because the existence of magnetic moment has not been discovered in Maxwell's time, the cognitive level based on Maxwell's time is not enough to support the use of magnetic moment to explain the origin of electric and magnetic fields)

Since we have explained the electric field from the aspect of the magnetic field, we need to explain the nature of magnetic and magnetic forces. For the nature of the magnetic force, we can assume from the kinetic point of view that the impulse of the angular momentum impinging on the photon of the medium causes the photon vortex to move, which in turn produces a medium impulse moment (similar to fan blowing). The medium impulse moment is the magnetic force, and the essence of the magnetic force line can be regarded as the cyclotron motion path of the medium 
photon. Thus, the magnetic force represents the cross product of the impulse $u$ applied by the electron to the photon of the medium with the vector of the photon displacement vector $r$, which can be expressed mathematically as:

$$
M=r \times u
$$

Magnetic field can be defined as "a vortex force system generated by photon cyclotron motion of electrons colliding with multiple spins", that is the superposition state of the magnetic moment. Its mathematical expression is:

$$
M=\sum m
$$

If the magnetic field line is used to express the magnetic field, then the magnetic field can also be expressed with the geometric changes of field strength and magnetic flux:

$$
\Phi_{H}=\iint_{s} H \cdot d s
$$

In this way, we have defined the nature of magnetic field and magnetic force from the perspective of mechanics, and thus unraveled the mystery about the intrinsic nature of electromagnetic field and electromagnetic force.

According to the law of Ampere Circle $\oint_{L} B \cdot d l=\mu_{0} \sum_{i=1}^{n} I_{i}$, the relationship between the magnetic field strength and the current in the current-carrying conductor can be expressed as:

$$
\oint_{L} H \cdot d l=\mu_{0} \sum_{i=1}^{n} I_{i}
$$

Taking into account that the field source of the magnetic field is the electron $e$, the force applied on static electrons in the magnetic field is:

$$
F=e H
$$

The force applied on moving electrons in the magnetic field is:

$$
F=e v \times H
$$

The total force acting on the current-carrying conductor in the magnetic field is:

$$
F=\int_{L} d F=\int_{L} I d l \times H
$$

Taking into account that the field source of the magnetic field is the electron $e$, and the impulse momentum produced by per unit electron is $M_{e}$. With reference to Biot-Savart law, it indicates that the physical meaning of current element is the electronic impulse moment $I d l \Rightarrow M_{e}$. Then the magnetic field strength generated by the electron at any point in the space can be expressed as:

$$
d H=k \frac{d M_{e} \times e_{r}}{r^{2}}=k \frac{I d l \times e_{r}}{r^{2}}
$$

The vector integral form is:

$$
H=\int_{L} d H=k \int_{L} \frac{d M_{e} \times e_{r}}{r^{2}}
$$

The magnetic interaction between two electrons can be expressed as: 


$$
d F_{1 \rightarrow 2}=k \frac{d M_{e 2} \times\left(d M_{e 1} \times e_{r}\right)}{r^{2}}
$$

When the vector is not considered, the acting forces between two electrons can be idealized as $F_{1 \rightarrow 2}=\frac{1}{4 \pi \varepsilon_{0}} \frac{e_{2} e_{1} r}{r^{3}}$. The equivalent Coulomb's law thus can be derived:

$$
F_{1 \rightarrow 2}=\frac{1}{4 \pi \varepsilon_{0}} \frac{e_{2} e_{1} r}{r^{3}} \Leftrightarrow \frac{1}{4 \pi \varepsilon_{0}} \frac{q_{2} q_{1} r}{r^{3}}
$$

It can be seen from the above that we can deduce the equivalent Coulomb's law from the angle of the magnetic field, and prove that the Coulomb electrostatic field is a current element magnetic field. Therefore, from the perspective of mechanics, we have proposed a feasible way to explain the electrostatic field with a magnetic field.

\section{Thus, we can come up with a prediction:}

"The frictional electrification phenomenon is the friction generating current (momentum flow) and the magnetic field".

\section{The mechanism is:}

Friction causes the electron to direct the collision motion to generate a momentum flow (current), and the electron momentum flow (current) polarizes the electron magnetic moment to generate a magnetic field (this is an electromagnet).

The rubbed object has the property of attracting light and small objects. The essence is that the friction generates a current, and the current acts on the electron magnetic moment of the small and small object, causing the electron magnetic moment to be polarized to display magnetic properties and generate a magnetic field. The attraction phenomenon is the interaction between two magnetic fields. Therefore, the frictional electrification phenomenon is the friction generating current and magnetic field.

\section{Therefore, we can draw 3 predictions:}

A: The rubbed ruler has a magnetic field that can cause small magnetic needle deflection.

B: The rubbed ruler has electromagnetic radiation.

C: The electromagnet can attract light and small objects (paper scraps).

\section{Experimental results:}

A: In the experiment, the rubbed ruler can trigger the deflection of the small magnetic needle (welcome to repeat this experiment), and the strength of the magnetic field generated by the rubbed ruler is $0.2 G s$.

B: The rubbed ruler has an electromagnetic radiation intensity of $0-3160 \mathrm{w} / \mathrm{cm}^{2}$.

Experimental video links ( $A$ and $B$ ):

https://www.ixigua.com/i6555401121496564231/

Note: Considering that the reviewers are busy, if the reviewers have to go and repeat the experiment in order to review a paper, this is particularly troublesome. So, I directly put the link of the 
experiment video here to facilitate the review. After passing the review, this link can be deleted for publication.

$\mathrm{C}$ : When the electromagnetic radiation value is $0-8181 \mathrm{w} / \mathrm{cm}^{2}$, the electromagnet can produce more intense and significant experimental phenomena of attracting shredded paper.

\section{Experimental verification method $(\mathbf{C})$ :}

We can confirm by observing whether there is an experimental phenomenon in the strong magnetic field that can attract small and light objects (crushed paper).

Discussion: If we see the phenomenon that a strong magnetic field can attract light and small paper scraps, the theory is correct. If we do not see such a phenomenon, the theory is wrong. (Although we have carried out experiments to verify our conjecture, we still invite you to verify our theory through repeated experiments).

Experimental equipment: power supply(AC), voltage regulator, after the power to generate magnetic field copper coil, paper, field strength measuring instrument

Experimental steps:

1: Series the power supply(AC), voltage regulator and copper coil

2: Sprinkle paper scraps around the copper coil

3: Turn on the power supply, through the voltage regulator to control the current, observe the copper coil around the magnetic field can attract paper scraps.

4: In the experiment, we can see that the greater the current, the greater the intensity of the magnetic field, attracting the more intense. the intensity of electromagnetic radiation (value range is $\left.0-8181 w / \mathrm{cm}^{2}\right)$.

This experiment proves that electromagnets (strong magnetic fields) can also attract shredded paper (or electrostatic attraction).

\section{Experimental video link:}

\section{https://www.ixigua.com/i6555403150042333710/}

Note: Considering that the reviewers are busy, if the reviewers have to go and repeat the experiment in order to review a paper, this is particularly troublesome. So, I directly put the link of the experiment video here to facilitate the review. After passing the review, this link can be deleted for publication.

\section{Discussion:}

According to the conclusions herein, the magnetic field can attract shredded paper. However, because ordinary magnets don't attract shredded paper and other light and small objects, I need to clarify. Here, the reason why ordinary magnets cannot attract shredded paper is that the magnetic force is small and the magnetization ability is weak. The rubbed ruler can attract shredded paper because friction generates current (momentum flow), current can re-arrange the electron magnetic 
moment of small and light objects and generate magnetic field, so the rubbed ruler is more likely to magnetize and attract paper scraps. (Note: Because the current can cause the electrons of light and small objects to be rearranged, and the electrons have magnetic moments, when the electrons are rearranged, the magnetic moment of the electrons will be rearranged again. Therefore, the magnetization ability of the current is stronger than that of ordinary permanent magnets. Can magnetize and attract shredded paper.).

In fact, because any object has an electronic magnetic moment, any object has an internal magnetic property. Therefore, in theory, as long as the magnetic field strength is strong enough, the magnet can attract any object. Therefore, we can use a powerful magnet to achieve the phenomenon of attracting shredded paper. In fact, powerful magnets can also attract hanging apples, tomatoes and kiwis, as well as attracting burnt matches (I can provide experimental videos).

\section{Experimental video link:}

https://www.ixigua.com/i6555404509365928455/

Note: Considering that the reviewers are busy, if the reviewers have to go and repeat the experiment in order to review a paper, this is particularly troublesome. So, I directly put the link of the experiment video here to facilitate the review. After passing the review, this link can be deleted for publication.

The force exerted by the current carry conductor $L_{1}$ on the electron $e_{2}$ is:

$$
d F_{1 \rightarrow 2}=M_{e 2} \times \int_{L_{1}} \frac{d M_{e 1} \times e_{r}}{r^{2}}
$$

The force exerted by the current carrying conductor $L_{1}$ on the current carrying conductor $L_{2}$ is:

$$
F_{1 \rightarrow 2}=\int_{L_{2}} d F_{1 \rightarrow 2}=\int_{L_{2}} \int_{L_{1}} \frac{d M_{e 2} \times\left(d M_{e 1} \times e_{r}\right)}{r^{2}}
$$

On the contrary:

$$
F_{L_{2} \rightarrow L_{1}}=-F_{L_{1} \rightarrow L_{2}}
$$

From the above, we can extrapolate that the magnetic field excited by the impulse of the electronic angular momentum follows the inverse square of the distance.

The moment of forces in the magnetic field can be expressed as:

$$
M=m \times H
$$

According to Faraday's law of electromagnetic induction, we can work out the relationship equation between EMF and magnetic field strength:

$$
\varepsilon_{i}=-\frac{d \Phi}{d t}=-\iint_{s} \frac{\partial H}{\partial t} \cdot d s
$$

It can be inferred from the above deduction that we can theoretically explain all the electric field phenomena from the aspect of the magnetic field, and predict that triboelectrification is a result of friction generating a magnetic field, which is another major breakthrough point in this paper.

Discussion: If triboelectrification is essentially a phenomenon where friction produces a magnetic field, then the unipolar electrostatic field is actually a dipole magnetic field. We can verify this by 
seeing if the charged scraps in the triboelectrification phenomenon have the magnetic properties of the two poles.

\section{A new expression model of Maxwell's equations at the micro level from the perspective of mechanics}

According to the above deduction, the origin of the electric field at the microscopic level can be understood as the electric current element magnetic field. Therefore, we need to give a new expression model of Maxwell's equations at the micro level from the perspective of mechanics.

According to the above-mentioned derivation of Faraday's law of electromagnetic induction, the electromotive force in the magnetic field is:

$$
\varepsilon_{i}=-\frac{d \Phi}{d t}=-\iint_{s} \frac{\partial H}{\partial t} \cdot d s
$$

The electromotive force $\varepsilon_{i}$ here represents the impulse force of the electron or the energy level difference of the electron (potential difference).

The fourth equation of Maxwell's equations is:

$$
\oint_{L} H \cdot d l=I_{o \text { int }}+\iint_{s} \frac{\partial D}{\partial t} \cdot d s
$$

Considering that the current density of electric displacement is $J_{d}=\frac{\partial D}{\partial t}$, and the current density of the conduction momentum flow is $J_{p}=\frac{d p}{d t \cdot d s_{\perp}}$, then the relationship between the electric displacement $D$ and electron momentum $p$ can be expressed as $\partial D=\partial p$, so the above equation can be improved as follows:

$$
\oint_{L} H \cdot d l=I_{o \text { int }}+\iint_{s} \frac{\partial p}{\partial t} \cdot d s
$$

This shows that changing electron momentum can generate a magnetic field.

Consider that the electric field is a manifestation of the magnetic field of the current element, and considering that the electric field and the magnetic field have the same field source, namely the electron $e$, the equations of the Maxwell's equations of the electric field and the magnetic field can be overlooked. Therefore, based on the new understanding at the micro level from the perspective of mechanics, the microscopic expression of Maxwell's equations from the perspective of mechanics is:

$$
\left\{\begin{array}{l}
\varepsilon_{i}=-\iint_{s} \frac{\partial H}{\partial t} \cdot d s \\
\oint_{L} H \cdot d l=I_{o \text { int }}+\iint_{s} \frac{\partial p}{\partial t} \cdot d s
\end{array}\right.
$$


It can be seen from the above derivation that from the perspective of mechanics that a changing magnetic field can generate an electromotive force, (electron impulse force or electron energy level difference or potential pressure), and a changing electron momentum can generate a magnetic field.

Discussion: We know that the initial form of equations proposed by Maxwell in 1865 consists of 20 equations and 20 variables. He tried to express it in quaternions in 1873, but was unsuccessful. The mathematical forms of the four equations used today were re-expressed in the form of vector analysis in 1884 by Oliver Heaviside and Josiah Gibbs. Today, based on the new understanding of the origin of charge, current, electric field, and magnetic field from the perspective of mechanics, we have further simplified the expression of Maxwell's equations from 4 equations to 2 equations. Therefore, this represents an advancement in physics. Of course, if you ask me whether we can further simplify the two equations of Maxwell's equations into one equation in the future? I think it is still possible.

\section{Conclusion}

In fact, many people familiar with electromagnetism know that this paper is not the first to use a mechanics analogy to electromagnetism. In history, many outstanding scientists have done similar research work, and their research work is even better than the expression in this article. There are only some problems that have not been resolved, that is, it has been unable to understand the origin of positive and negative charges from the perspective of mechanics, and how the electric and magnetic fields are generated by electric charges from the perspective of mechanics. This article just solves this problem. Therefore, this article realizes an analogy understanding of the conceptual foundations of electromagnetics from the perspective of mechanics.

In other words, this article tries to understand the origin of charge, current, voltage, resistance, electric field and magnetic field from the perspective of mechanics to satisfy my childhood curiosity. I found that from the perspective of mechanics that charge can be understood as a form of electron momentum, current can be understood as a momentum flow, voltage can be understood as the potential pressure or energy level difference of the electron orbital potential, resistance can be understood as a momentum resistance, and magnetic field can be understood as the superposition state of magnetic moments, the electric field can be understood as a manifestation of the magnetic field of the current element. In this way, I satisfied my childhood curiosity and realized my wish for many years.

\section{Acknowledgement}

First of all, I thank my good brother Hao-yang Zhang from the Department of Physics of Oxford University for the discussion.

Secondly, I would like to thank the Nobel Prize winner in 1973, Professor Josephson of the Cavendish Laboratory of the University of Cambridge, for the one-month discussion of my paper and his valuable suggestions for my experiment.

Finally, I am especially grateful to the founder of classical electrodynamics, one of the greatest physicists of mankind, James Clerk Maxwell, for enlightening me on electromagnetics. You are my source of exploration of nature and the beacon of spiritual freedom. 


\section{References}

1. Eringen A C, Maugin G A. Microscopic Electromagnetic Theory[M]// Electrodynamics of Continua I. Springer New York, 1990.

2. Shadid W G T. Two New Theories for the Current Charge Relativity and the Electric Origin of the Magnetic Force Between Two Filamentary Current Elements[J]. IEEE Access, 2016, 4:4509-4533.

3. Ivezic T. Nature of Electric and Magnetic Fields; How the Fields Transform[J]. Physics, 2015.

4. Ioffe A Z D A F. Origin of the Magnetic Field[C]// International Astronomical Union Colloquium. Cambridge University Press, 1986.

5. Ferris M R, Gratus J. The origin of the Schott term in the electromagnetic self-force of a classical point charge[J]. Journal of Mathematical Physics, 2011, 52(9):092902.

6. Qi X L, Li R, Zang J, et al. Inducing a Magnetic Monopole with Topological Surface States[J]. Science, 323.

7. Niemi A J, Paranjape M B, Semenoff G W. Electric Charge of the Magnetic Monopole[J]. Phys.rev.lett, 1984, 53(53):515-518.

8. Bhattacharya R. Comment on the CP-violating electric charge of the magnetic monopole[J]. Zeitschrift Für Physik C Particles \& Fields, 1986, 32(2):215-220.

9. Kazama Y, Yang C N, Goldhaber A S. Scattering of a Dirac particle with charge Z e by a fixed magnetic monopole[J]. Physical Review D, 1976, 15:8(15):2287-2299.

10. Goldhaber A S, Singh H, Parwani R. On the fractional electric charge of a magnetic monopole at nonzero temperature[J]. Physics Letters B, 1996, 386(1-4):207-210.

11. Jaubert L D C, Holdsworth P C W. Magnetic monopole dynamics in spin ice[J]. Journal of Physics: Condensed Matter, 2011, 23(16):164222.

12. Sivers, Dennis. Possible Binding of a Magnetic Monopole to a Particle with Electric Charge and a Magnetic Dipole Moment[J]. Physical Review D, 1970, 2(9):2048-2054.

13. Davidson A, Guendelman E I. Electric monopole with internal magnetic-monopole-like structure[J]. Physics Letters B, 1990, 251(2):250-253.

14. $\sim$ Halliday D, $\sim$ Resnick R, Walker J. Fundamentals of Physics[J]. Physics Today, 2003, 25(4):53-54.

15. Ashcroft $\mathrm{N}$ W, Mermin N D. SOLID STATE PHYSICS[M]. HOLT, RINEHART AND WINSTON, 1976.

16. Datz S, Drake G W F, Gallagher T F, et al. Atomic physics[J]. Rev.mod.phys, 1999, 71(2):377-407.

17. Elton, B L R. Quantum Mechanics (Non-Relativistic Theory) [J]. Physics Bulletin, 1958, 9(10):270-271.

18. Scully M O, Zubairy M S, Milonni P W. Quantum Optics[J]. Physics Today, 1998, 51(10):90-92. 